\title{
ON SEPARATION OF LATTICES
}

MABEL SZETO

Department of Mathematics

College of Staten Island

The City University of New York

130 Stuyvesant Place

Staten Island, New York 10301

(Received June 6, 1990 and in revised form October 4, 1990)

\begin{abstract}
Separation properties of a lattice of subsets of an arbitrary set or separation properties between a pair of such lattices have strong implications on the associated lattice regular measures and conversely. A number of such relationships are investigated and applications given to topological lattices in a topological space.
\end{abstract}

KEY WORDS AND PHRASES. Disjunctive and normal lattices, separation properties, associated lattice regular measures, lattice separation and semi-separation.

1980 AMS SUBJECT CLASSIFICATION CODE. 28C15, 54D35, 54060.

\section{(I) Introduction}

In the first part of this paper we consider various separation properties pertaining either to a single lattice $\mathcal{L}$ of subsets of an arbitrary set $X$ or to a pair of such lattices $\mathcal{L}_{1}$ and $\dot{L}_{2}$. These separation properties will have strong implications pertaining to the associated lattice regular measures. and in the particular case of $0-1$ valued lattice regular measures, these separation properties will have associated filter and topological implications.

In the second part of the paper we consider the converse situation and obtain results which imply various lattice separations under assumptions on the behaviour of the associated lattice regular measures.

The results of both parts can be utilized to obtain many specific topological separations by appropriately choosing the lattices in given topological spaces.

The approach will be measure-theoretic throughout since we will be concerned with extensions and restrictions of measures and in the case of $0-1$ valued measures for which one could use analogous filter arguments, the measure arguments are simpler and more natural in this setting, and more importantly, hold with minor modifications for non $0-1$ valued measures for which there is no filter analogue.

In the next section we will review briefly the notations and definitions used throughout this paper. The terminology is consistent with that of [1]. [3]. [T]. [S] and others. We then consider lattice separations and some of its measure ind topological consequences in sections 3 and 4 , and finally in section 5 , we consider measure relations which imply separation conditions between lattices and other lattice conditions. 


\section{(II) Background and notation}

We collect here the notations and definitions that will be used throughout and supply: a bit of background material. so that the paper will be reasonably self-contained.

Let $\mathcal{L}$ be a lattice of subsets of an abstract set $X$. We assume throughout that 0 . $I \in \mathcal{L}$. We denote by:

(1) $\mathcal{A}(\mathcal{L})$ the algebra generated by $\mathcal{L}$.

(2) $\sigma(\mathcal{L})$ the $\sigma$-algebra generated by $\mathcal{L}$.

(3) $\delta(\mathcal{L})$ the lattice of all countable intersections of sets from $\mathcal{L}$.

(4) $\tau(\mathcal{L})$ the lattice of all arbitrary intersections of sets from $\mathcal{L}$.

(5) $s(\mathcal{L})$ the lattice derived Souslin sets.

We denote by $M(\mathcal{L})$ those finite valued, non-negative, finitely additive measures on $\mathcal{A}(\dot{\mathcal{L}})$ which are non-trivial. $M_{\sigma}(\mathcal{L})$ denotes those $\mu \in M(\mathcal{L})$ which are $\sigma$-smooth on $\mathcal{L}$. i.e.. $L_{n} \in \mathcal{L}, L_{n} \downarrow \phi$, then $\mu\left(L_{n}\right) \rightarrow 0$.

If $\mu \in M(\mathcal{L})$. then $\mu$ is $\mathcal{L}$-regular if for any $A \in \mathcal{A}(\mathcal{L}), \mu(\mathcal{A})=\sup \{\mu(L) \mid L \subset A, L \in$ $\mathcal{L}$ \}. We denote by $M_{R}(\mathcal{L})$, the set of all $\mathcal{L}$-regular measures of $M(\mathcal{L})$. By $M_{R}^{\sigma}(\mathcal{L})$ we mean those $\mu \in M_{R}(\mathcal{L})$ which are $\sigma$-smooth on $\mathcal{L}$. Clearly, if $\mu \in M_{R}^{\sigma}(\mathcal{L})$, then $\mu$ is $\sigma$-smooth on $\mathcal{A}(\mathcal{L})$. and is, consequently, countably additive.

$M^{\sigma}(\mathcal{L})$ denotes those $\mu \in M(\mathcal{L})$, which are countably additive on $\mathcal{A}(\mathcal{L})$.

We denote by $I(\mathcal{L}), I_{\sigma}(\mathcal{L}), I_{R}(\mathcal{L}), I^{\sigma}(\mathcal{L})$ the two-valued $0-1$ measures of $. M(\mathcal{L}) . M_{\sigma}(\mathcal{L})$. $M_{R}(\mathcal{L})$ and $M^{\sigma}(\mathcal{L})$ respectively. It is not difficult to show that there is a one-to-one correspondence between:

$I(\mathcal{L})$ and all prime $\mathcal{L}$-filters

$I_{R}(\mathcal{L})$ and all $\mathcal{L}$-ultrafilters

$I_{\sigma}(\mathcal{L})$ and all prime $\mathcal{L}$-filters with the countable intersection property

$I_{R}^{\sigma}(\mathcal{L})$ and all $\mathcal{L}$-ultrafilters with the countable intersection property.

We next note some standard lattice terminology; $\mathcal{L}$ is called:

a) complemented if $L \in \mathcal{L}$ implies $L^{\prime} \in \mathcal{L}$ (where prime denotes complement), that is. $\mathcal{L}$ is an algebra.

b) separating if for any two elements $x_{1}, x_{2} \in X, x_{1} \neq x_{2}$ there exists an element $L \in \mathcal{L}$ such that $x_{1} \in L$ and $x_{2} \notin L$.

c) $T_{2}$ if. for any two elements $x_{1} \neq x_{2}$ of $X$. there exists $L_{1}, L_{2} \in \mathcal{L}$ such that $x_{1} \in L_{1}^{\prime}, x_{2} \in L_{2}^{\prime}$ and $L_{1}^{\prime} \cap L_{2}^{\prime}=\phi$. 
d) drsjunctive if for $x \in X$ and $L \in \mathcal{L}$ and such that $x \notin L$. there exists an $A \in \mathcal{L}$ with $x \in A$, and $A \cap L=\varphi$.

e) normal if for $L_{1}, L_{2} \in \mathcal{L}$ and $L_{1} \cap L_{2}=0$. there exists $\mathcal{A}, B \in \mathcal{L}$ with $L_{1} \subset A^{\prime} . L_{2} \subset B^{\prime}$. and $A^{\prime} \cap B^{\prime}=0$.

f) delta lattzce $(\delta$-lattice $)$ if $\delta(\mathcal{L})=\mathcal{L}$.

g) compact lattice if for any collection $\left\{L_{\alpha}\right\}$ of sets of $\mathcal{L} . \cap L_{\alpha}=0$ implies there exists a finite subcollection with an empty intersection.

Similarly we define $\mathcal{L}$ countably compact (also called semi-compact or quasicompact by some writers) and $\mathcal{L}$-regular. We note that there are equivalent measure characterization for all these properties. In order to state these, we first define the support of $\mu \in M(\mathcal{L})$, denoted by $S(\mu)=\cap\left\{L \in \mathcal{L} \mid \mu(L)=\mu\left(X^{-}\right)\right\}$. Hence, if $\mu \in I(\mathcal{L})$, then $S(\mu)=\cap\{L \in \mathcal{L}|| \mu(L)=1\}$.

If $\mu_{1}, \mu_{2} \in M(\mathcal{L})$. we write $\mu_{1} \leq \mu_{2}(\mathcal{L})$ when $\mu_{1}(L) \leq \mu_{2}(L)$ for all $L \in \mathcal{L}$.

It is then easy to show

(1) $\mathcal{L}$ is complemented if and only if $I(\mathcal{L})=I_{R}(\mathcal{L})$. (See section $\jmath^{3}$ )

(2) $\mathcal{L}$ is separating if and only if for $x, y \in X, x \neq y, y \notin S\left(\mu_{x}\right)$ and $x \notin S\left(\mu_{y}\right)$ where $\mu_{x}$ is the measure concentrated at $x$. i.e. $\mu_{x}(A)=1$ if and only if $x \in A$, where $A \in \mathcal{A}(\mathcal{L})$.

(3) $\mathcal{L}$ is $T_{2}$ if and only if for any $\mu \in I(\mathcal{L}) . S(\mu)=\phi$ or a singleton.

(4) $\mathcal{L}$ is disjunctive if and only if $\mu_{x} \in I_{R}(\mathcal{L})$ for all $x \in X$.

(5) $\mathcal{L}$ is regular if and only if $\mu_{1} \leq \mu_{2}(\mathcal{L}), \mu_{1}, \mu_{2} \in I(\mathcal{L})$ implies $S\left(\mu_{1}\right)=S\left(\mu_{2}\right)$.

(6) $\mathcal{L}$ is normal if and only if for any $\mu \in I(\mathcal{L})$ if $\mu \leq \nu_{1}(\mathcal{L})$ and $\mu \leq \nu_{2}(\mathcal{L})$ (we recall that $\mu \leq \nu_{1}(\mathcal{L})$ if $\mu(L) \leq \nu_{1}(L)$ for all $\left.L \in \mathcal{L}\right)$ and $\nu_{1}, \nu_{2} \in I_{R}(\mathcal{L})$ implies $\nu_{1}=\nu_{2}$.

(7) $\mathcal{L}$ is compact if and only if $S(\mu) \neq \phi$ for any $\mu \in I_{R}(\mathcal{L})$ : equivalently for any $\mu \in I(\mathcal{L})$.

(8) $\mathcal{L}$ is countably compact if and only if $I_{R}(\mathcal{L})=I_{R}^{\sigma}(\mathcal{L})$; equivalently $I(\mathcal{L})=$ $I_{\sigma}(\mathcal{L})$.

It is possible to go on in this manner. but the above should indicate the useful interplay between measure and topological concepts.

The proofs of all the above are quite short; typically we prove:

(4): If $\mathcal{L}$ is disjunctive, let $L^{\prime} \in \mathcal{L}^{\prime}$, the complement lattice, and if $\mu_{x}\left(L^{\prime}\right)=1$, then, 
by disjunctiveness, there exists an $A \in \mathcal{L}$, with $x \in A$. o $\mu_{x}(A)=1$, and $A \subset L^{\prime}$. It follows immediately that $\mu_{x} \in I_{R}(\mathcal{C})$. The converse is clear.

We assume throughout that $\mathcal{L}$ is disjunctive. Let $A \in \mathcal{A}(\mathcal{L})$, and define $\Psi^{\circ}(\mathcal{A})=\{\mu \in$ $\left.I_{R}(\mathcal{L}) \mid \mu(\mathcal{A})=1\right\}$. Then it is clear that

(1) $W^{-}(A \cup B)=W(A) \cup W(B)$

(2) $W^{*}(A \cap B)=W^{*}(A) \cap W^{\circ}(B)$

(3) $\quad W^{\circ}\left(A^{\prime}\right)=W^{-}(A)^{\prime}$

(4) $W(A) \subset W(B)$ if and only if $A \subset B$.

Take $I_{R}(\mathcal{L})$ with $W(\mathcal{L})=\{W(L) \mid L \in \mathcal{L}\}$ as a base for the closed sets of a topology. This is the Wallman space with the Wallman topology: It is easily seen to be $T_{1}$, and is compact. The compactness follows immediately if we just show $\boldsymbol{W}^{-}(\mathcal{L})$ is a compact lattice. Let $\nu \in I_{R}\left(W^{\prime}(\mathcal{L})\right)$, then $\nu\left(W^{\prime}(\mathcal{A})\right)=\hat{\mu}\left(W^{-}(\mathcal{A})\right)$, where in general $\hat{\mu}\left(W^{*}(A)\right)$ is defined for $\mu \in I_{R}(\mathcal{L})$ as follows:

$$
\hat{\mu}(W(A))=\mu(A) .
$$

This gives a bijective correspondence between $I_{R}(\mathcal{L})$ and $I_{R}\left(I^{-}(\mathcal{L})\right.$ ). Finally,

$$
S(\nu)=S(\hat{\mu})=\cap\{W(L) \mid \hat{\mu}(W(L))=1\},
$$

but $\hat{\mu}\left(\mathscr{W}^{-}(L)\right)=1$ is equivalent to $\mu \in W^{*}(L)$. Hence, $\mu \in S(\nu)$ and this completes the proof.

We note that if. in addition, $\mathcal{L}$ is separating, then the map $x \rightarrow \mu_{x}$ is an embedding of $X$ into $I_{R}(\mathcal{L})$ such that $\bar{X}=W(X)=I_{R}(\mathcal{L})$. If $X$ is given the $\tau(\mathcal{L})$ topology it is easily seen to be a homeomorphic embedding.

Specific topological lattices give rise to various well-known compactifications of $X$ :

(1) If $X$ is a $T_{3 \frac{1}{2}}$ space and $\mathcal{L}=\mathcal{Z}$, the lattice of zero sets, then $I_{R}(\mathcal{Z})=\beta X$, the Stone-Cech compactification.

(2) If $X$ is a $T_{1}$ topological space and $\mathcal{L}=\mathcal{F}$, the lattice of closed sets, then $I_{R}(\mathcal{F})=\omega X$, the Wallman compactification.

(3) If $X$ is a zero-dimensional $T_{2}$ space and $\mathcal{L}=\mathcal{C}$, the lattice of clopen sets, then $I_{R}(\mathcal{C})=\beta_{0} X$, the Banachewski compactification.

Other topological cases will be cited later in the examples. If $\mathcal{L}=\mathcal{L}^{\prime}$; i.e., if $\mathcal{L}$ is an algebra, then $I_{R}(\mathcal{L})=I(\mathcal{L}), W(L)^{\prime}=W\left(L^{\prime}\right) \in W(\mathcal{L})$ for $L \in \mathcal{L}$, and we obtain the Stone space associated with the algebra $\mathcal{L}$. 
Finilly, we list for reference the following two theorems:

THEORLM 2.1. If $\mathcal{L}_{1}, \mathcal{L}_{2}$ are lattices of subsets of $X$ such that $\mathcal{L}_{1} \subset \mathcal{L}_{2}$. then any $\mu \in$ $M_{l i}\left(\mathcal{L}_{1}\right)$ can be extended to a $\nu \in M_{R}\left(\mathcal{L}_{2}\right)$. If $\mathcal{L}_{1}$ semiseparate $\mathcal{L}_{2}$, then the extension will alway's be unique if and only if $\mathcal{L}_{1}$ rparates $\mathcal{L}_{2}$ (i.e., $A, B \in \mathcal{L}_{2}, A \cap B=\phi$ inplies there exist $C, D \in \mathcal{L}_{1}$ such that $A \subset C, B \subset D$. and $\left.C \cap D=\delta\right)$. If $\mu \in M M_{R}^{\sigma}\left(\mathcal{L}_{1}\right)$ and if $\mathcal{L}_{1}$ is a $\delta$-lattice, and $\mathcal{L}_{2}$ is $\sigma\left(\mathcal{L}_{1}\right)$ bounded or just $\mathcal{S}_{\mu^{*}}$ bounded. then $\nu^{\prime} \in M_{R}^{\sigma}\left(\mathcal{L}_{2}\right)$. (We say in general for any two lattices $\mathcal{L}_{1}$ and $\mathcal{L}_{2}$ that $\mathcal{L}_{2}$ is $\mathcal{L}_{1}$ bounded if $B_{n} \in \mathcal{L}_{2}$ for all $n$. $B_{n} \downarrow \phi$ implies there exist $A_{n} \in \mathcal{L}_{1}$ such that $\left.B_{n} \subset A_{n} \downarrow \phi\right)$. Also, $\mathcal{S}_{\mu}$. denotes the $\mu^{*}$-measurable sets (See [2]).

TIIEOREM 2.2. Let $\mathcal{L}$ be a $\delta$-lattice of subsets of $X$ such that $\sigma(\mathcal{L}) \subset s(\mathcal{L})$. then every $\mu \in M^{\sigma}(\mathcal{L})$ is $\mathcal{L}$-regular, i.e.. $M_{R}^{\sigma}(\mathcal{L})=M^{\sigma}(\mathcal{L})$.

This theorem follow's inımediately from the Choquet capacity theorem.

\section{(III) On normal lattices}

Let $X$ be an abstract set and $\mathcal{L}$ a lattice of subsets of $X$ such that $\phi$ and $X$ belong to $\mathcal{L}$. It was noted in section 2 that $W(\mathcal{L})$ is a $T_{1}$ lattice and that $I_{R}(\mathcal{L})$ with $W(\mathcal{L})$ as a base for the closed subsets (the Wallman topology) is a compact $T_{1}$ space.

We note that $W^{\prime}(\mathcal{L})$ is always disjunctive, for suppose $\mu \notin W(A)$ where $A \in \mathcal{L}$. Then $\mu\left(A^{\prime}\right)=1$ and there exists $B \in \mathcal{L}, B \subset A^{\prime}$ with $\mu(B)=1$. Thus $\mu \in W^{\top}(B)$ and $W(A) \cap W(B)=W(A \cap B)=\phi$.

Although the following theorem is generally known we show for completeness:

Theorem 3.1. If $\mathcal{L}$ is disjunctive, then $I_{R}(\mathcal{L})$ with the Wallman topology is $T_{2}$ if and only if $\mathcal{L}$ is normal.

Proof: If $\mathcal{L}$ is normal, then it is easy to ser that $W(\mathcal{L})$ is normal. Consequently $W^{-}(\mathcal{L})$ is $T_{1}$, disjunctive, and normal and therefore $T_{2}$ from which it follows that the Wallman topology is $T_{2}$.

Conversely, suppose the topology is $T_{2}$. Then $W(\mathcal{L})$ is $T_{2}$. Let $\mu \in I(\mathcal{L})$ and consider $\hat{\mu} \in I(W(\mathcal{L}))$, then there exists a unique $\nu \in S(\hat{\mu})$, and consequently a unique $\nu \in I_{R}(\mathcal{L})$ with $\mu \leq \nu(\mathcal{L})$.

Since $W(\mathcal{L})$ is always disjunctive and compact $T_{1}$, one can show 
(1) $W^{*}(\mathcal{L})$ is normal

(2) $W^{\prime}(\mathcal{L})$ is regular

(3) $\boldsymbol{H}^{-}(\mathcal{L})$ is $T_{2}$.

Proor: If (1) holds, then $\mathscr{I}^{-}(\mathcal{L})$ is normal and dinjunctive and therefore clearly regular. While if (2) holds, then $\mathbb{I}^{-}(\mathcal{L})$ is regular and $T_{1}$ and therefore $T_{2}$. Finally: if $\mathbb{I}^{-}(\mathcal{L})$ is $T_{2}$. then since it is compact. it must be normal.

We observe a few further facts about normal lattices which are of interest in their own right. but which will not be used in the subsequent topological applications.

Again let $X$ be an abstract set with $\mathcal{L}$ an arbitrary lattice of subsets containing $\phi$ and $\mathrm{X}$, and let $\mu \in I(\mathcal{L})$. For any $E \subset \mathbb{X}$. define $\mu^{\prime}(E)=\inf \left\{\mu\left(L^{\prime}\right): E \subset L^{\prime} . L \in \mathcal{L}\right\}$. Clearly $\mu^{\prime}$ is a finitely subadditive outer measure. and $\mu \leq \mu^{\prime}(\mathcal{L})$ and $\mu=\mu^{\prime}$ on $\mathcal{L}$ if and only if $\mu \in I_{R}(\mathcal{L})$. Now we show

ThEORE.M 3.3. If $\mathcal{L}$ is normal. then $\mathcal{G}=\left\{L \in \mathcal{L}: \mu^{\prime}(L)=1\right\}$ is an $\mathcal{L}$-ultrafilter.

Proof: Clearly $\phi \notin \mathcal{G}$ and $\mathcal{G}$ is closed under $\mathcal{L}$-supersets. Now suppose $\mathcal{L}_{1}, \mathcal{L}_{2} \in \mathcal{G}$. then $\mu^{\prime}\left(L_{1}\right)=1=\mu^{\prime}\left(L_{2}\right)$. If $\mu^{\prime}\left(L_{1} \cap L_{2}\right)=0$, then there exists an $L \in \mathcal{L}$ such that $L_{1} \cap L_{2} \subset L^{\prime}$ and $\mu\left(L^{\prime}\right)=0$. so $\mu(L)=1$. But $L \subset L_{1}^{\prime} \cup L_{2}^{\prime}$ and since $\mathcal{L}$ is normal. $L=A_{1} \cup A_{2}$ where $A_{1} \subset L_{1}^{\prime} . A_{2} \sqsubset L_{2}^{\prime}$ and $A_{1} . A_{2} \in \mathcal{L}$. Since $\mu(L)=1$. we have $\mu\left(A_{1}\right)=1$ or $\mu\left(A_{2}\right)=1$. If $\mu\left(A_{1}\right)=1$, then $A_{1}^{\prime} \supset L_{1}$ and we get $\mu^{\prime}\left(L_{1}\right)=0$. a contradiction. Similarly $\mu\left(A_{2}\right)=1$ leads to a contradiction. Thus $\mu^{\prime}\left(L_{1} \cap L_{2}\right)=1$, and $\mathcal{G}$ is an $\mathcal{L}$-filter. Suppose $\mathcal{G} \subset \mathcal{H}$ where $\mathcal{H}$ is an $\mathcal{L}$-filter. and $L \in \mathcal{H}$ with $L \notin \mathcal{G}$. Then $\mu^{\prime}(L)=0$. Hence $L \subset A^{\prime} . A \in \mathcal{L}$ and $\mu\left(A^{\prime}\right)=0$. Therefore $\mu(A)=1, \mu^{\prime}(A)=1$, and $A \in \mathcal{G} \subset \mathcal{H}$, but $A \cap L=0$. a contradiction. Hence $\mathcal{G}$ is an $\mathcal{L}$-ultrafilter.

If $\nu \in I_{R}(\mathcal{L})$ goes with the $\mathcal{L}$-ultrafilter $\mathcal{G}$ (see section 2), then $\mu \leq \nu(\mathcal{L})$. Thus if $\mathcal{L}$ is normal, to each $\mu \subseteq I(\mathcal{L})$, we can construct a $\nu \in I_{R}(\mathcal{L})$ such that $\mu \leq \nu(\mathcal{L})$ directly without recourse to Zorn's lemma. We can also prove the uniqueness of $\nu$ quite simply from this For if $\mu \leq \backslash(\mathcal{L})$ where $\lambda \in I_{R}(\mathcal{L})$, then $\lambda \leq \mu=\mu^{\prime}\left(\mathcal{L}^{\prime}\right)$. Suppose $\lambda(\mathcal{A})=1$ where $A \equiv \mathcal{L}$. and $\mu^{\prime}(A)=0$. Then $A \subset B^{\prime}, B \in \mathcal{L}$ and $\mu\left(B^{\prime}\right)=0$. so $\lambda\left(B^{\prime}\right)=0$. but then $\lambda(A)=0$. a contradiction. Hence $\lambda(A)=1$ implies $\mu^{\prime}(A)=\nu(A)=1$. and $\lambda \leq \nu(\mathcal{L})$. so $\lambda=\nu$. 
In the more general case of $\mu \in M(\mathcal{L}), \mu \geq 0$, we must proceed in a slightly more complicated way since there is no $\mathcal{L}$-filter or $\mathcal{L}$-ultrafilter to assist us. For $\mu \in M(\mathcal{L})$, $\mu \geq 0$, we define for $E \subset X, \lambda(E)=\sup \{\mu(L): L \subset E, L \in \mathcal{L}\}$. If $\mathcal{L}$ is normal, then $\lambda$ is finitely subadditive on $\mathcal{L}^{\prime}$ : namely let $A, B \in \mathcal{L}$ and $L \subset A^{\prime} \cup B^{\prime}$ where $L \in \mathcal{L}$. then $L=L_{1} \cup L_{2}$ where $L_{1}, L_{2} \in \mathcal{L}$ and $L_{1} \subset A^{\prime}, L_{2} \subset B^{\prime}$. Now $\lambda(L)=\mu(L)=\mu\left(L_{1} \cup L_{2}\right) \leq$ $\mu\left(L_{1}\right)+\mu\left(L_{2}\right)$, but $\mu\left(L_{1}\right)+\mu\left(L_{2}\right)=\lambda\left(L_{1}\right)+\lambda\left(L_{2}\right) \leq \lambda\left(A^{\prime}\right)+\lambda\left(B^{\prime}\right)$ from which it follows that $\lambda\left(A^{\prime} \cup B^{\prime}\right) \leq \lambda\left(A^{\prime}\right)+\lambda\left(B^{\prime}\right)$. Next, for $E \subset X$, let $\hat{\mu}(E)=\inf \left\{\lambda\left(L^{\prime}\right): E \subset L^{\prime} . L \in \mathcal{L}\right\}$. It is now routine to show that $\hat{\mu}$ is a finitely subadditive outer measure. Now we show

(3.1) $E \subset X$ is $\hat{\mu}$-measurable if and only if

$$
\hat{\mu}\left(A^{\prime}\right) \geq \hat{\mu}\left(A^{\prime} \cap E\right)+\hat{\mu}\left(A^{\prime} \cap E^{\prime}\right) \text { for all } A \in \mathcal{L} .
$$

Proof: Let $S \subset X$ be arbitrary and let $A \in \mathcal{L}$ with $S \subset A^{\prime}$, then

$$
\begin{aligned}
\lambda\left(A^{\prime}\right)=\hat{\mu}\left(A^{\prime}\right) & \geq \hat{\mu}\left(A^{\prime} \cap E\right)+\hat{\mu}\left(A^{\prime} \cap E^{\prime}\right) \\
& \geq \hat{\mu}(S \cap E)+\hat{\mu}\left(S \cap E^{\prime}\right)
\end{aligned}
$$

from which we get $\hat{\mu}(S) \geq \hat{\mu}(S \cap E)+\hat{\mu}\left(S \cap E^{\prime}\right)$ and we are clearly done.

Next we have

(3.2) Every element of $\mathcal{L}^{\prime}$ is $\hat{\mu}$-measurable.

Proof: Let $B^{\prime} \in \mathcal{L}^{\prime}$, then by $(3.1)$ we must show that $\hat{\mu}\left(A^{\prime}\right) \geq \hat{\mu}^{\prime}\left(A^{\prime} \cap B^{\prime}\right)+\hat{\mu}\left(A^{\prime} \cap B\right)$ for all $A^{\prime} \in \mathcal{L}^{\prime}$. Let $D \in \mathcal{L}$ such that $D \subset A^{\prime} \cap B^{\prime}$, and let $F \in \mathcal{L}$, and $F \subset A^{\prime} \cap D^{\prime}$, so $D \cap F=\phi$, and $D \cup F \subset A^{\prime}$. Then $\hat{\mu}\left(A^{\prime}\right)=\lambda\left(A^{\prime}\right) \geq \mu(D \cup F)=\mu(D)+\mu(F)$, and this immediately implies that

$$
\hat{\mu}\left(A^{\prime}\right) \geq \hat{\mu}\left(A^{\prime} \cap B^{\prime}\right)+\hat{\mu}\left(A^{\prime} \cap B\right) .
$$

From (3.2) it follows that $\mathcal{A}(\mathcal{L}) \subset \mathcal{S}_{\hat{\mu}}=$ the $\hat{\mu}$-measurable sets, and that $\hat{\mu}$ restricted to $\mathcal{A}(\mathcal{L})$ (which we continue to denote by $\hat{\mu}$ ) is in $M_{R}(\mathcal{L})$ since for $L^{\prime} \in \mathcal{L}^{\prime}, \hat{\mu}\left(L^{\prime}\right)=\lambda\left(L^{\prime}\right)=$ $\sup \{\mu(A): A \subset L, A \in \mathcal{L}\}$. Also it is clear that $\hat{\mu}(X)=\mu(X)$, and $\mu \leq \hat{\mu}(\mathcal{L})$.

Suppose there exists a $\nu \in M_{R}(\mathcal{L})$ such that $\mu(X)=\nu(X)$, and $\mu \leq \nu(\mathcal{L})$, then $\mu \leq \hat{\mu} \leq \hat{\nu}=\nu(\mathcal{L})$, but since both $\hat{\mu}$ and $\nu$ belong to $M_{R}(\mathcal{L})$ and $\hat{\mu}(X)=\nu(X)$, it follows that $\mu=\nu$. In summary we have

THEOREM 3.4. If $\mathcal{L}$ is a normal lattice of subsets of $X$, and if $\mu \in M(\mathcal{L})$, then there exists a unique $\nu \in M_{R}(\mathcal{L})$ such that $\mu \leq \nu(\mathcal{L})$ and $\mu(X)=\nu(X)$. 
REMARK: In a similar manner, one can show that if $\mathcal{L}_{1}$ and $\mathcal{L}_{2}$ are lattices of subsets of $I$ such that $\mathcal{L}_{1} \subset \mathcal{L}_{2}$, and $\phi, X \in \mathcal{L}_{1}$, and if for any $A \subset B^{\prime} \cup C^{\prime}, A \in \mathcal{L}_{1}, B . C \in \mathcal{L}_{2}$, there exists $A_{1}, A_{2} \in \mathcal{L}_{1}$ such that $A=A_{1} \cup A_{2}, A_{1} \subset B^{\prime}, A_{2} \subset C^{\prime}$, then any $\mu \in M_{R}\left(\mathcal{L}_{1}\right)$ can be uniquely extended to a $\nu \in M_{R}\left(\mathcal{L}_{2}\right)$, and $\nu$ is actually $\mathcal{L}_{1}$-regular on all sets of $\mathcal{L}_{2}^{\prime}$. Related material can be found in [4].

\section{(IV) Lattice separation}

Suppose that $\mathcal{L}_{1}$ and $\mathcal{L}_{2}$ are lattices of subsets of an abstract set $X$.

Definition 4.1: $\mathcal{L}_{1}$ semi-separates $\mathcal{L}_{2}$ if $A \cap B=\phi, A \in \mathcal{L}_{1}, B \in \mathcal{L}_{2}$ implies there exists a $C \in \mathcal{L}_{1}$ such that $B \subset C$, and $A \cap C=\phi . \mathcal{L}_{1}$ separates $\mathcal{L}_{2}$ if $B_{1}, B_{2} \in \mathcal{L}_{2}$, and $B_{1} \cap B_{2}=0$ implies there exists $A_{1}, A_{2} \in \mathcal{L}_{1}$ such that $B_{1} \subset A_{1}, B_{2} \subset A_{2}$, and $A_{1} \cap A_{2}=\phi$.

We assume now that $\mathcal{L}_{1} \subset \mathcal{L}_{2}$ and $\phi, X \in \mathcal{L}_{1}$, and although it is generally known (see [2]). for convenience, we prove:

LEMMA 4.1. If, as above, $\mathcal{L}_{1} \subset \mathcal{L}_{2}$ and $\mathcal{L}_{1}$ semi-separates $\mathcal{L}_{2}$, then for any $\nu \in M_{R}\left(\mathcal{L}_{2}\right)$. the restriction $\mu$ of $\nu$ to $\mathcal{A}\left(\mathcal{L}_{1}\right)$ belongs to $M_{R}\left(\mathcal{L}_{1}\right)$.

Proof: Consider any $A^{\prime}$ where $A \in \mathcal{L}_{1}$. Since $\nu \in M_{R}\left(\mathcal{L}_{2}\right)$. for any $\epsilon>0$, there exists $B \in \mathcal{L}_{2}$ such that $B \subset A^{\prime}$ and

$$
\mu\left(A^{\prime}\right)=\nu\left(A^{\prime}\right) \leq \nu(B)+\epsilon .
$$

Then, by semi-separation, there is a $C \in \mathcal{L}_{1}$ with $B \subset C \subset A^{\prime}$, so $\mu\left(A^{\prime}\right) \leq \nu(B)+\epsilon \leq$ $\nu(C)+\epsilon=\mu(C)+\epsilon$, and it follows easily that $\mu \in M_{R}\left(\mathcal{L}_{1}\right)$.

Under the assumptions of Lemma 4.1, we have, in particular, that for any $\nu \in I_{R}\left(\mathcal{L}_{2}\right)$, the restriction $\mu$ of $\nu$ to $\mathcal{A}\left(\mathcal{L}_{1}\right)$ belongs to $I_{R}\left(\mathcal{L}_{1}\right)$. Hence we have a mapping $\psi: I_{R}\left(\mathcal{L}_{2}\right) \rightarrow$ $I_{R}\left(\mathcal{L}_{1}\right)$ where, for $\nu \in I_{R}\left(\mathcal{L}_{2}\right), \psi(\nu)=\mu$ is the restriction of $\nu$ to $\mathcal{A}\left(\mathcal{L}_{1}\right)$. It is easy to see that $\psi$ is continuous with respect to the Wallman topologies on $I_{R}\left(\mathcal{L}_{2}\right)$ and $I_{R}\left(\mathcal{L}_{1}\right)$ respectively. Moreover, $\psi$ is onto since, in general, any $\mu \in M_{R}\left(\mathcal{L}_{1}\right)$ can be extended to a $\nu \in M_{R}\left(\mathcal{L}_{2}\right)$ such that $\mu(X)=\nu(X)$, and if $\mu \in I_{R}\left(\mathcal{L}_{1}\right)$ one can find a $\nu \in I_{R}\left(\mathcal{L}_{2}\right)$ (see [2]). We note that in the case of $0-1$ valued measures that any $\mu \in I_{R}\left(\mathcal{L}_{1}\right)$ can be extended to a $\nu \in I_{R}\left(\mathcal{L}_{2}\right)$ can be established by simple lattice filter arguments.

Finally, we note that if $\mathcal{L}_{1}$ separates $\mathcal{L}_{2}$, then it is easy to see that $\psi$ is homeomorphism. Consequently, if $\mathcal{L}_{1}$ separates $\mathcal{L}_{2}$ and if either $I_{R}\left(\mathcal{L}_{1}\right)$ or $I_{R}\left(\mathcal{L}_{2}\right)$ is $T_{2}$, then so is the other. 
but assuming disjunctiveness, this is equivalent to normality of the particular lattice as was shown in Theorem 3.1 and we have:

THEOREM 4.1. If $\mathcal{L}_{1}$ and $\mathcal{L}_{2}$ are disjunctive lattices of subsets of $\mathrm{X}$ such that $\phi, X \in \mathcal{L}_{1}$ where $\mathcal{L}_{1} \subset \mathcal{L}_{2}$ and if $\mathcal{L}_{1}$ separates $\mathcal{L}_{2}$, then $\mathcal{L}_{1}$ is normal $\Leftrightarrow I_{R}\left(\mathcal{L}_{1}\right)$ is $T_{2} \Leftrightarrow I_{R}\left(\mathcal{L}_{2}\right)$ is $T_{2} \Leftrightarrow \mathcal{L}_{2}$ is nom mal.

We now give several applications of these results.

(1) Let $X$ be a $T_{3 \frac{1}{2}}$ topological space and $\mathcal{F}$ the lattice of closed subsets, and $\mathcal{Z}$ the lattice of zero sets. $I_{R}(\mathcal{F})=\omega X$, the Wallman compactification of $X$, and $I_{R}(\mathcal{Z})=3 . X$. the Stone-Cech compactification of $X$. Then if $\beta X=\omega X, \omega \cdot X$ is $T_{2}$ and, therefore $X$ is normal by Theorem 3.1. Conversely, if $X$ is normal, then $\mathcal{Z}$ separates $\mathcal{F}$ and $I_{R}(\mathcal{Z})$ is homeomorphic to $I_{R}(\mathcal{F})$ via the map $\psi$, and in general, in the disjunctive case, $\psi^{\prime}\left(\mu_{x}\right)=\mu_{x}$, using $\mu_{x}$ again for the restriction, where $\mu_{x}$ is the measure concentrated at $x$, so $\beta X=\omega X$.

Similarly, we have, denoting the regular closed sets of $X$ by $\mathcal{F}_{R}$ and the generated $\delta$-lattice by $\delta\left(\mathcal{F}_{R}\right)$, that $I_{R}\left(\delta\left(\mathcal{F}_{R}\right)\right)=\beta X$ implies that $\delta\left(\mathcal{F}_{R}\right)$ is normal. Conversely. if $\mathcal{Z}$ separates $\delta\left(\mathcal{F}_{R}\right)$, then $I_{R}\left(\delta\left(\mathcal{F}_{R}\right)\right)=\beta \mathrm{X}$, and $\delta\left(\mathcal{F}_{R}\right)$ is normal.

(2) Since a 0-dimensional topological space is strongly 0-dimensional if and only if $\mathcal{C}$, the lattice of clopen sets separates $\mathcal{Z}$, the lattice of zero sets, we obtain similarly that $\beta_{0} X=\beta . X$ if and only if the 0 -dimensional space $X$ is strongly 0 -dimensional, where $\beta_{0} X=I_{R}(\mathcal{C})$ is the Banaschewski compactification of $X$.

(3) Since, in general, the regular open sets $\mathcal{O}_{R}$ separates the open sets, we also obtain that $I_{R}\left(\mathcal{O}_{R}\right)=I_{R}(\mathcal{O})$, where we view $\mathcal{O}_{R}$ as the lattice generated by, the regular open sets. If in addition, $X$ is 0 -dimensional and extremally disconnected, then $\mathcal{C}$ separates $\mathcal{O}$. and in this case $I_{R}(\mathcal{O})=\beta_{0} X$. It is not difficult to show that $I_{R}(\mathcal{O})$ can be identified with the Stone space $I(R(X))$, where $R(X)$ is the complete Boolean algebra of regular closed sets of $X$ with $\vee A_{\alpha}=\overline{\left(\cup A_{\alpha}^{\circ}\right)}$ and $\wedge A_{\alpha}=\overline{\left(\cap A_{\alpha}\right)^{\circ}}$, for $A_{\alpha} \in R(X)$. It follows immediately from well-known facts about the Stone space that $I(R(X))$ and, therefore, $I_{R}(\mathcal{O})$ is extremally disconnected.

(4) Recall that a $T_{1}$ space $X$ is called seminormal if $\mathcal{F}_{R}$, the lattice generated by the regular closed sets, separates $\mathcal{F}$. We then have $I_{R}\left(\mathcal{F}_{R}\right)=\omega X$, the Wallman compactification if $X$ is seminormal. The space $I_{R}\left(\mathcal{F}_{R}\right)$ is the same as $\omega_{k} X$, defined by Ponomarev and studied by Zaicév. 
(5) Finally, we note that if $X$ is a regular $T_{1}$ space, then $X$ is quasinormal if $\mathcal{F}_{R}$, the lattice generated by the regular closed sets is normal. We immediately get from the theorem that if $X$ is quasinormal and seminormal, then $X$ is normal. We observe that if $X$ is a $T_{3 \frac{1}{2}}$ space, it is known that $\omega_{k} X=\beta X$ if and only if $X$ is quasinormal. Consequently: if $\mathrm{X}$ is also seminormal, then $\omega X=\omega_{k} X=\beta X$.

We note that part of Theorem 4.1 can be extended to nondisjunctive lattices, namely:

THEOREM 4.2. If $\mathcal{L}_{1}$ and $\mathcal{L}_{2}$ are lattices of subsets of $X$-uch that $\phi, X \in \mathcal{L}_{1}$ where $\mathcal{L}_{1} \subset \mathcal{L}_{2}$ and if $\mathcal{L}_{1}$ separates $\mathcal{L}_{2}$, then $\mathcal{L}_{1}$ is normal if and only if $\mathcal{L}_{2}$ is normal.

PROOF: If $\mu \in I\left(\mathcal{L}_{1}\right)$ and if $\mu \leq \nu_{1}\left(\mathcal{L}_{1}\right)$ and $\mu \leq \nu_{2}\left(\mathcal{L}_{1}\right)$ where $\nu_{1}, \nu_{2} \in I_{R}\left(\mathcal{L}_{1}\right)$. Now $\mu$ extends to a $\lambda \in I\left(\mathcal{L}_{2}\right)$ and $\nu_{1}, \nu_{2}$ extend to $\tau_{1}, \tau_{2} \in I_{R}\left(\mathcal{L}_{2}\right)$ respectively. We show that $\lambda \leq \tau_{1}\left(\mathcal{L}_{2}\right)$. Suppose there exists $L_{2} \in \mathcal{L}_{2}$ such that $\lambda\left(L_{2}\right)=1$, but $\tau_{1}\left(L_{2}\right)=0$. Then $\tau_{1}\left(L_{2}^{\prime}\right)=1$ and since $\tau_{1} \in I_{R}\left(\mathcal{L}_{2}\right)$, there exists an $A_{2} \subset L_{2}^{\prime}, A_{2} \in \mathcal{L}_{2}$ and $\tau_{1}\left(A_{2}\right)=1$. Since $\mathcal{L}_{1}$ separates $\mathcal{L}_{2}$, there is an $L_{1} \in \mathcal{L}_{1}$ such that $L_{2} \subset L_{1} \subset A_{2}^{\prime}$. Then $\mu\left(L_{1}\right)=1$. so $1=\nu_{1}\left(L_{1}\right)=\tau_{1}\left(L_{1}\right)$, and $\tau_{1}\left(A_{2}^{\prime}\right)=1$, a contradiction. Thus $\lambda \leq \tau_{1}\left(\mathcal{L}_{2}\right)$. and similarly $\lambda \leq \tau_{2}\left(\mathcal{L}_{2}\right)$. Hence if $\mathcal{L}_{2}$ is normal $\tau_{1}=\tau_{2}$ and therefore $\nu_{1}=\nu_{2}$ and $\mathcal{L}_{1}$ is normal. The converse follows immediately by using the fact that the restrictions of elements of $I_{R}\left(\mathcal{L}_{2}\right)$ are in $I_{R}\left(\mathcal{L}_{1}\right)$ and are distinct.

Definition 4.2: If $\mathcal{L}_{1}$ and $\mathcal{L}_{2}$ are lattices of subsets of $X$, then $\mathcal{L}_{1}$ coseparates $\mathcal{L}_{2}$ if $A \cap B=\phi . A . B \in \mathcal{L}_{2}$ implies there exists $C, D \in \mathcal{L}_{1}$ such that $A \subset C^{\prime}, B \subset D^{\prime}$, and and $C^{\prime} \cap D^{\prime}=\phi$.

In a normal topological space $X$, the lattice of closed sets $\mathcal{F}$ coseparates itself, as does the lattice of zero sets in a $T_{3 \frac{1}{2}}$ space. Any algebra of subsets of a set $X$ clearly coseparates itself.

The following is easy to prove:

THEOREM 4.3. Let $\mathcal{L}_{1}$ and $\mathcal{L}_{2}$ be lattices of subsets of $X$.

(1) If $\mathcal{L}_{1}$ coseparates $\mathcal{L}_{2}$, then for any $A \subset B^{\prime} \cup C^{\prime}, A \in \mathcal{L}_{1}, B, C \in \mathcal{L}_{2}$, there exist $A_{1}, A_{2} \in \mathcal{L}_{1}$ such that $A=A_{1} \cup A_{2}, A_{1} \subset B^{\prime}, A_{2} \subset C^{\prime}$ (see remark after Theorem 4.1).

(2) If $X \in \mathcal{L}_{1}$, the converse of statement (1) holds.

(3) If $\phi, X \in \mathcal{L}_{1} \subset \mathcal{L}_{2}$, then if $\mathcal{L}_{1}$ coseparates $\mathcal{L}_{2}, \mathcal{L}_{1}$ separates $\mathcal{L}_{2}$. 


\section{(V) On semiseparation}

We noted in section 4 that if $\mathcal{L}_{1} \subset \mathcal{L}_{2}$, and if $\mathcal{L}_{1}$ semiseparates $\mathcal{L}_{2}$ then $\psi: I_{R}\left(\mathcal{L}_{2}\right) \rightarrow$ $I_{R}\left(\mathcal{L}_{1}\right)$, where $\psi$ is the restriction map. In this section we consider partial converses of this statement. We denote the basic closed sets of $I_{R}\left(\mathcal{L}_{1}\right)$ with a subscript 1 , such as $W_{1}\left(L_{1}\right)$ where $I_{1} \in \mathcal{L}_{1}$, and those of $I_{R}\left(\mathcal{L}_{2}\right)$ with a subscript 2 , such as $W_{2}\left(L_{2}\right), L_{2} \in \mathcal{L}_{2}$.

If $\psi^{*}: I_{R}\left(\mathcal{L}_{2}\right) \rightarrow I_{R}\left(\mathcal{L}_{1}\right)$, and if $\mathcal{L}_{2}$ is disjunctive then clearly $\mathcal{L}_{1}$ must also be disjunctive, since $\psi \mu_{x} \in I_{R}\left(\mathcal{L}_{1}\right)$ where $\mu_{x}$ is the measure concentrated at $x \in \mathrm{X}$. We continue to denote this restriction by $\mu_{x}$. Also, if $\psi: I_{R}\left(\mathcal{L}_{2}\right) \rightarrow I_{R}\left(\mathcal{L}_{1}\right)$, then $\psi$ is continuous with respect to the Wallman topologies since $\psi^{-1}\left(W_{1}\left(L_{1}\right)\right)=W_{2}\left(L_{2}\right)$, where $L_{1} \in \mathcal{L}_{1}$.

LEMMA 5.1. If $\mathcal{L}_{2}$ is disjunctive and if $\mathcal{L}_{1}$ is normal and if $\psi: I_{R}\left(\mathcal{L}_{2}\right) \rightarrow I_{R}\left(\mathcal{L}_{1}\right)$ where $\mathcal{L}_{1} \subset \mathcal{L}_{2}$, then $\psi\left(W_{2}\left(L_{2}\right)\right)=\cap\left\{W_{1}\left(L_{1 \alpha}\right) \mid L_{2} \subset L_{1 \alpha}, L_{1 \alpha} \in \mathcal{L}_{1}\right\}$ where $L_{2} \in \mathcal{L}_{2}$.

Proof: Since $W_{2}\left(L_{2}\right)$ is closed, it is compact. Therefore $\psi\left(W_{2}\left(L_{2}\right)\right)$ is compact, and therefore, closed since $\mathcal{L}_{1}$ is normal. Hence,

$$
\psi\left(W_{2}\left(L_{2}\right)\right)=\cap W_{1}\left(L_{1 \alpha}\right)
$$

where the $L_{1 \alpha} \in \mathcal{L}_{1}$, and it follows by the disjunctiveness of $\mathcal{L}_{2}$ that $L_{2} \subset L_{1 \alpha}$.

We now have:

THEOREM 5.1. Let $\mathcal{L}_{1} \subset \mathcal{L}_{2}$ be lattices of subsets of $X$. If $\mathcal{L}_{2}$ is disjunctive and $\mathcal{L}_{1}$ is normal, and if $\psi: I_{R}\left(\mathcal{L}_{2}\right) \rightarrow I_{R}\left(\mathcal{L}_{1}\right)$, then $\mathcal{L}_{1}$ semiseparates $\mathcal{L}_{2}$.

Proof: Suppose $L_{2} \in \mathcal{L}_{2}$ and $L_{1} \in \mathcal{L}_{1}$ and $L_{2} \cap L_{1}=\phi$. Then $W_{2}\left(L_{2}\right) \cap W_{2}\left(L_{1}\right)=\phi$. Consequently, $\psi\left(W_{2}\left(L_{2}\right)\right) \cap W_{1}\left(L_{1}\right)=\phi$, for if $\mu \in W_{1}\left(L_{1}\right)$, and if $\mu=\psi(\nu)$ where $\nu \in W_{2}\left(L_{2}\right)$, then $\nu\left(L_{2}\right)=1$ and $\nu\left(L_{1}\right)=\mu\left(L_{1}\right)=1$, a contradiction. Thus $\psi\left(W_{2}\left(L_{2}\right)\right) \cap$ $W_{1}\left(L_{1}\right)=\phi$, and by Lemma 5.1, $\cap\left\{W_{1}\left(L_{1 \alpha}\right) \mid L_{2} \subset L_{1 \alpha}, L_{1 \alpha} \in \mathcal{L}_{1}\right\} \cap W_{1}\left(L_{1}\right)=\phi$, and by the compactness of $W_{1}\left(\mathcal{L}_{1}\right)$, we get

$$
\bigcap_{i=1}^{n} W_{1}\left(L_{1 \alpha_{1}}\right) \cap W_{1}\left(L_{1}\right)=\phi
$$

but then

$$
L_{2} \subset \bigcap_{i=1}^{n} L_{1 \alpha_{i}}=L \in \mathcal{L}_{1},
$$

and $L \cap L_{1}=\phi$ which proves that $\mathcal{L}_{1}$ semiseparates $\mathcal{L}_{2}$. As an immediate consequence, we have 
COROLLARY 5.1. Let $\mathcal{L}_{1} \subset \mathcal{L}_{2}$ be lattices of subsets of $X$. If $\mathcal{L}_{2}$ is disjunctive and countably compact. and if $\mathcal{L}_{1}$ is $\delta$-normal, and if $\sigma\left(\mathcal{L}_{1}\right) \subset s\left(\mathcal{L}_{1}\right)$, then $\mathcal{L}_{1}$ semiseparates $\mathcal{L}_{2}$.

ProOF : Let $\nu \in I_{R}\left(\mathcal{L}_{2}\right)=I_{R}^{\sigma}\left(\mathcal{L}_{2}\right)$ since $\mathcal{L}_{2}$ is countably compact. Now $\psi(\nu)=\mu \in I^{\sigma}\left(\mathcal{L}_{2}\right)$ and by Choquet's theorem (see section 2), we have that $\mu \in I_{R}^{\sigma}\left(\mathcal{L}_{1}\right)$ which, by Theorem j.1. completes the proof.

Another immediate consequence, which is easy to prove, is:

COROLlary 5.2. Let $\mathcal{L}_{1} \subset \mathcal{L}_{2}$ be lattices of subsets of $X$ where $\mathcal{L}_{2}$ is disjunctive and $\mathcal{L}_{1}$ is normal. If $\psi: I_{R}\left(\mathcal{L}_{2}\right) \rightarrow I_{R}\left(\mathcal{L}_{1}\right)$ is a homeomorphism, then $\mathcal{L}_{1}$ separates $\mathcal{L}_{2}$.

We note that if $\dot{\mathcal{L}}_{1}$ and $\mathcal{L}_{2}$ are both separating and disjunctive lattices so that $X$ is densely embedded in both $I_{R}\left(\mathcal{L}_{1}\right)$ and $I_{R}\left(\mathcal{L}_{2}\right)$ (see section 2), and if $\mathcal{L}_{1} \subset \mathcal{L}_{2}$, then if $f: I_{R}\left(\mathcal{L}_{2}\right) \rightarrow I_{R}\left(\mathcal{L}_{1}\right)$ is a homeomorphism leaving $X$ fixed, we can, again assuming $\mathcal{L}_{1}$ is normal and using standard denseness arguments, show that $f=\psi$.

As another application of Theorem 5.1, we note that if $\mathcal{L}$ is a lattice of subsets of $X$ such that $I(\mathcal{L})=I_{R}(\mathcal{L})$, then $\mathcal{L}$ is clearly normal. Now if $\mathcal{L}_{2}$ is an algebra of sets containing $\mathcal{L}$, then $\mathcal{L}_{2}$ is, of course, disjunctive. Moreover, $\psi: I_{R}\left(\mathcal{L}_{2}\right)=I\left(\mathcal{L}_{2}\right) \rightarrow I(\mathcal{L})=I_{R}(\mathcal{L})$. Therefore, $\mathcal{L}$ semiseparates $\mathcal{L}_{2}$. But, $L^{\prime} \in \mathcal{L}_{2}$ where $L \in \mathcal{L}$; hence, there exists an $A \in \mathcal{L}$ such that $L^{\prime} \subset A$, and $A \cap L=\phi$. so $L^{\prime}=A \in \mathcal{L}$, and $\mathcal{L}$ is complemented, i.e. $\mathcal{L}=\mathcal{L}^{\prime}$. This results holds true even if $\mathcal{L}$ is just an abstract distributive lattice (see [3] and [6]).

COROLla RY 5.3. If $\mathcal{L}$ is a lattice of subsets of $X$ such that $I(\mathcal{L})=I_{R}(\mathcal{L})$, then $\mathcal{L}=\mathcal{L}^{\prime}$.

Definition 5.1: If $\mathcal{L}_{1}$ and $\mathcal{L}_{2}$ are lattices of subsets of $X, \mathcal{L}_{2}$ is $\mathcal{L}_{1}$-bounded if $B_{n} \downarrow \phi$, $B_{n} \in \mathcal{L}_{2}$ implies that there exists $A_{n} \in \mathcal{L}_{1}, B_{n} \subset A_{n}$ for all $n$ and $A_{n} \downarrow \phi$.

As a supplement to Corollary 5.1 , we have

THEOREM 5.2. If $\mathcal{L}_{1} \subset \mathcal{L}_{2}$ are lattices of subsets of $X$, then

a) If $\mathcal{L}_{2}$ is disjunctive and countably compact and if $\mathcal{L}_{1}$ is $\delta$-normal, and $\sigma\left(\mathcal{L}_{1}\right) \subset$ $s\left(\mathcal{L}_{1}\right)$, then $\mathcal{L}_{1}$ semiseparates $\mathcal{L}_{2}$ and, of course, $\mathcal{L}_{1}$ is countably compact.

b) If $\mathcal{L}_{2}$ is $\mathcal{L}_{1}$-bounded and if $\mathcal{L}_{1}$ is countably compact. then $\mathcal{L}_{2}$ is countably compact.

Proof: a) This is just Corollary 5.1 . 
b) Lat $\nu \in I\left(\mathcal{L}_{2}\right)$, and let $\mu$ be the restriction of $\nu$ to $\mathcal{A}\left(\mathcal{L}_{1}\right)$. Then $B_{n} \downarrow \varphi$. $B_{n} \in \mathcal{L}_{2}$ implies that there exist $A_{n} \in \mathcal{L}_{1}, B_{n} \subset A_{n} \downarrow \phi$ so $\nu\left(B_{n}\right) \leq \nu\left(A_{n}\right)=\mu\left(A_{n}\right) \rightarrow 0$, since $\mu \in I\left(\dot{\mathcal{L}}_{1}\right)=I_{\sigma}\left(\mathcal{L}_{1}\right)$. Therefore. $I\left(\mathcal{L}_{2}\right)=I_{\sigma}\left(\mathcal{L}_{2}\right)$, and $\mathcal{L}_{2}$ is count ably compact.

As a special case of this theorem, we get

COROLlaRY 5.4. Let $X$ be a completely regular $T_{2}$ topological space. Then

a) If $X$ is countably compact. then $X$ is pseudocompact and $\mathcal{Z}$, the lattice of zero sets semiseparates $\mathcal{F}$, the lattice of closed sets.

b) If $\mathrm{X}$ is a pseudocompact $c b$-space, then $\mathrm{X}$ is countably compact.

Proof: The proof is immediate from Theorem 5.2, taking $\mathcal{L}_{2}=\mathcal{F}$, and $\mathcal{L}_{1}=\mathcal{Z}$.

The corollary is well-known, and we note that it has also been shown that in $c b$-spaces. $\mathcal{Z}$ semiseparates $\mathcal{F}$. To proceed in this spirit, we must replace $I_{R}(\mathcal{L})$ and $W^{-}(\mathcal{L})$ in our discussion by $I_{R}^{\sigma}(\mathcal{L})$ and $W_{\sigma}(\mathcal{L})=W^{\top}(\mathcal{L}) \cap I_{R}^{\sigma}(\mathcal{L})$, and use the fact that if $\mathcal{L}$ is disjunctive, then $W_{\sigma}(\mathcal{L})$ is a replete lattice. We do not pursue these matters here since they take us out of the main spirit of this paper.

Finally, it is clear, as in section 4 , that many other applications of these results can be given for other specific choices of $\mathcal{L}_{1}$ and $\mathcal{L}_{2}$ as topological lattices.

\section{References}

1. Alexandroff, A.D., Additive set functions in abstract spaces. Recueil Mathematique T. 8 (50) \#2 (1940), pp. 307-342; T.9 (51) \#3 (1941), pp. 563-621.

2. Bachman, G., and A. Sultan, On extensions of regular measures, Pacific J. Math., 86, No. 2 (1980), pp. 389-395.

3. Bourbaki, N., Elements of Mathematics, General Topology, part 1, AddisonWeseley, 1966.

4. Camacho, J. Jr., Extensions of lattice regular measures with applications, Journal of the Indian Math. Soc., Vol. 54 (1989), pp. 233-244.

5. Grassi, P., An adjoint map between regular measures and its applications, Journal of the Indian Math. Soc., Vol. 48 (1984), pp. 19-31. 
6. Huerta, C.C., Measure requirements on distributive lattices for Boolean algebras and topological applications, Proceedings of the American Mathematical Society, Vol. 106, No. 2, June 1989, pp. 30i-308.

7. Nöbeling, G., Grundlagen der analytischen Topologie, Springer, Berlin. 1954.

8. Wallman, H., Lattices and topological spaces, Ann. of Math., 39 (1938), pp. 112-126. 


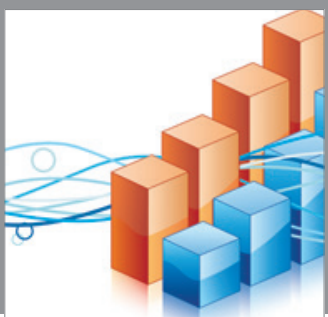

Advances in

Operations Research

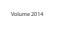

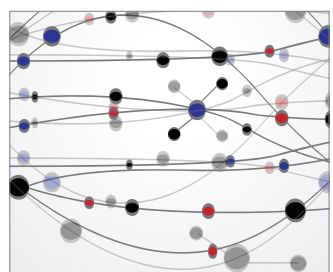

\section{The Scientific} World Journal
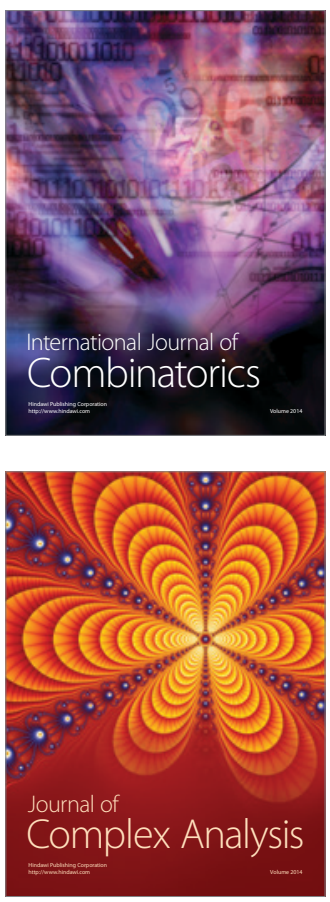

International Journal of

Mathematics and

Mathematical

Sciences
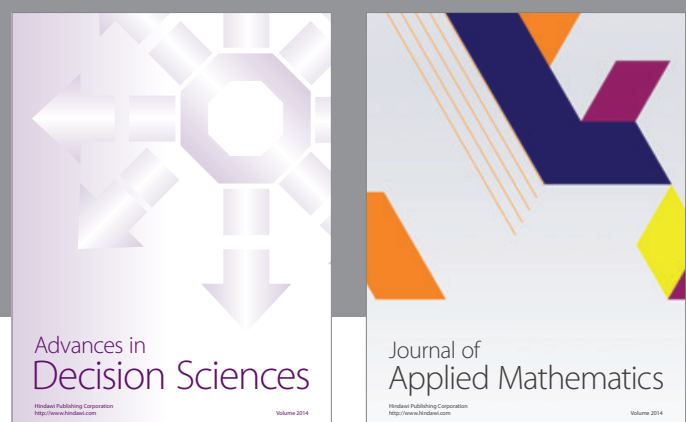

Journal of

Applied Mathematics
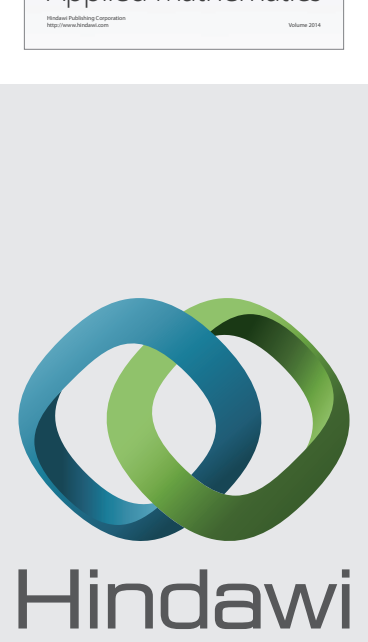

Submit your manuscripts at http://www.hindawi.com
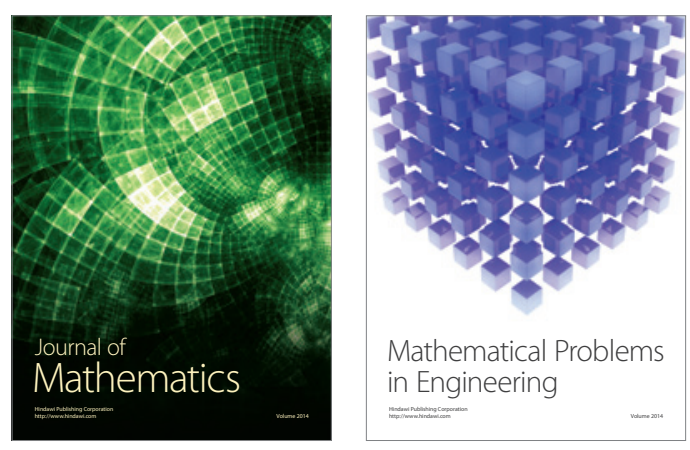

Mathematical Problems in Engineering
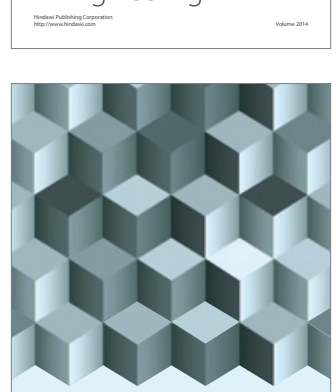

Journal of

Function Spaces
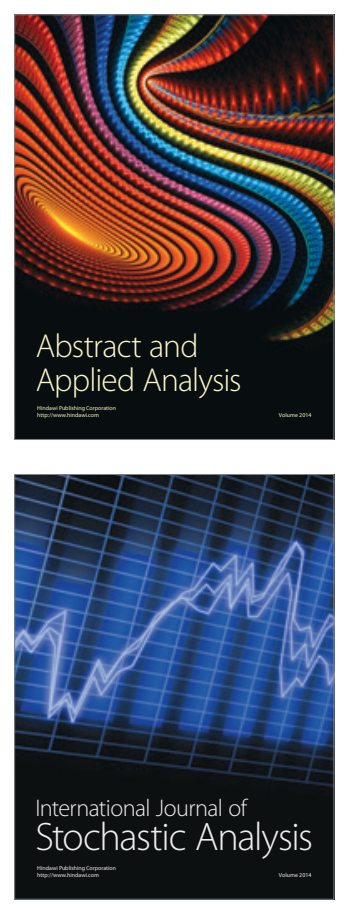

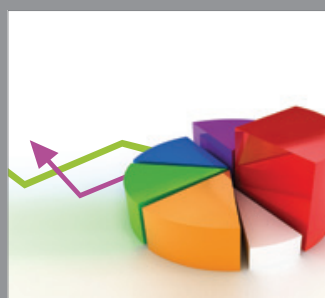

ournal of

Probability and Statistics

Promensencen
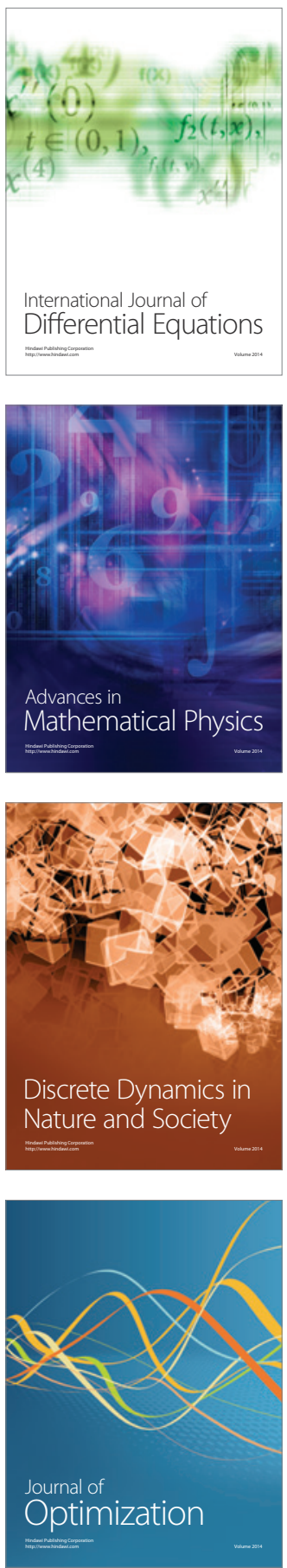